Received: 15 March 2017

Accepted: 29 August 2017

Published online: 11 September 2017

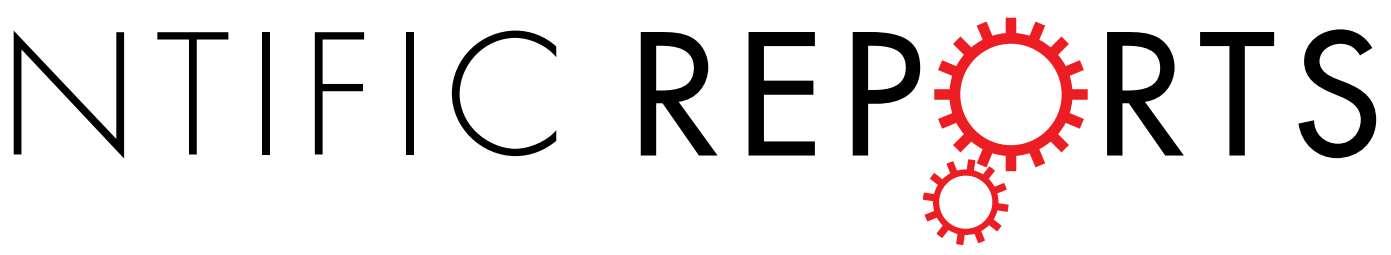

\title{
OPEN Idiopathic male infertility and polymorphisms in the DNA methyltransferase genes involved in epigenetic marking
}

Qiuqin Tang ${ }^{1}$, Yiqiu Chen ${ }^{2,3}$, Wei Wu ${ }^{2,3,4}$, Hongjuan Ding ${ }^{1}$, Yankai Xia ${ }^{2,3}$, Daozhen $C^{2}{ }^{4} \&$ Xinru Wang ${ }^{2,3}$

The purpose of this study was to investigate the association between male infertility and singlenucleotide polymorphisms (SNPs) of DNA methyltransferases (DNMT) genes (DNMT3B: rs2424909, DNMT1: rs4804490, DNMT3A: rs1550117 and DNMT3L: rs7354779). Eight hundred and thirty three idiopathic infertile males and four hundred and ten fertile controls from the hospitals affiliated to Nanjing Medical University between 2010 and 2012 were recruited in the study. We demonstrated a significantly increased risk of idiopathic infertility with abnormal semen parameters in association with the heterozygous genotype of variant rs 4804490 . Moreover, the AA genotype of variant rs 4804490 was associated with significantly decreased risk for male infertility with abnormal semen parameters. A decreased risk of idiopathic infertility with abnormal semen parameters was associated with the homozygous genotype of variant rs2424909. These results suggested that variants in different $D N M T$ genes have different relationships with idiopathic male infertility, and Chinese men carrying these variants have an increased or decreased risk of abnormal semen parameters.

Male infertility is a heterogeneous disorder that contributes to the impairment of spermatogenesis. Clinical investigations have confirmed that a significant proportion of male infertility is idiopathic, the molecular mechanisms underlying the defects have not been elucidated ${ }^{1,2}$. Most studies published to date support the view that abnormal epigenetic changes also resulted in male infertility. Lande-Diner and other researchers have concluded that DNA methylation is an essential epigenetic modification specifically related to gene silencing ${ }^{3}$. Aberrant DNA methylation has been proposed as a possible mechanism compromising male fertility ${ }^{4-6}$. The aberrant DNA methylation found in infertile males may be a consequence of a dysfunction in the machinery involved in establishing and maintaining normal DNA methylation. Accumulating evidence has indicated that male infertility results from gene mutations and single-nucleotide polymorphisms (SNP) $)^{7,8}$.

Mouse models have shown that aberrant DNA methylation patterns resulting from gene targeting of the DNMTs may cause spermatogenic defects ${ }^{9-12}$. DNA methyltransferases (DNMTs) are a family of proteins responsible for transferring methyl groups specifically to cytosine in CpG dinucleotides ${ }^{13,14}$. Genes involved in the epigenetic pathways are likely to affect DNA methylation and are associated with male infertility. Researchers have analyzed the genetic variants within the genes to investigate the epigenetic processes in the aetiology of diseases ${ }^{15}$.

Four DNMTs $\left(D N M T 1^{16}, D N M T 3 A, D N M T 3 B^{17-19}\right.$, and $\left.D N M T 3 L^{20,21}\right)$ have been extensively characterized in mammals. DNMT1, which is often referred to as the maintenance methyltransferase, is responsible for maintaining pre-existing methylation patterns during DNA replication ${ }^{22,23}$. DNMT3A and DNMT3B are considered to be de novo DNA methyltranferases, which are critical in the dynamic DNA methylation process during

\footnotetext{
${ }^{1}$ State Key Laboratory of Reproductive Medicine, Department of Obstetrics, Nanjing Maternity and Child Health Hospital, Obstetrics and Gynecology Hospital Affiliated to Nanjing Medical University, Nanjing, 210004, China. ${ }^{2}$ State Key Laboratory of Reproductive Medicine, Institute of Applied Toxicology, School of Public Health, Nanjing Medical University, Nanjing, 211166, China. ${ }^{3}$ Key Laboratory of Modern Toxicology of Ministry of Education, Nanjing Medical University, Nanjing, 211166, China. ${ }^{4}$ Clinical Laboratory, Wuxi Maternity and Child Health Care Hospital Affiliated to Nanjing Medical University, Wuxi, 214002, China. Qiuqin Tang and Yiqiu Chen contributed equally to this work. Correspondence and requests for materials should be addressed to W.W. (email: wwu@njmu.edu.cn) orY.X. (email: yankaixia@njmu.edu.cn)
} 
embryogenesis and pathogenesis ${ }^{24}$. In addition to these active enzymes, the DNMT3L protein, catalytically inactive by itself, also contributes to de novo methylation by interacting with the catalytic domains of DNMT3A and $D N M T 3 B$ and enhancing their enzymatic activity ${ }^{25-27}$. DNA methylation, mediated by DNMTs, is required for proper embryonic development ${ }^{18,28}$ and for the formation of mature functional germ cells $s^{9,10,29}$. Dysregulation of the DNMTs may lead to various conditions ${ }^{30}$, including spermatogenic defects ${ }^{12}$, autoimmune disorders ${ }^{31}$ and cancer ${ }^{13,32}$. To better understand the relationships between polymorphisms in DNMTs and idiopathic male infertility, we studied the associations between genetic variation in DNMT genes and altered semen parameters. We show that variants in the DNMT3B and DNMT1 may have different relationships with idiopathic male infertility. Furthermore, we compared the transcriptional factors between C allele and T allele of rs $2424909, \mathrm{C}$ allele and the A allele of rs4804490.

\section{Materials and Methods}

Subject recruitment and sample collection. The study was approved by the Institutional Ethics Committee of Nanjing Medical University. All activities involving human subjects were done under full compliance with government policies and the Helsinki Declaration. The participants wrote informed consents prior to the study. All study subjects were consecutively recruited from the Affiliated Hospitals of Nanjing Medical University between 2010 and 2012 (NJMU Infertility Study). The inclusion criteria consisted of five terms: (i) a normal 46, XY karyotype; (ii) absence of Y chromosomal microdeletions of AZF region; (iii) lack of hypogonadotropic hypogonadism; (iv) normal sexual and ejaculatory functions and no seminal tract obstruction, varicocele; (v) no history of infection or other diseases that could affect fertility. Finally, 833 men are included as the study subjects. The controls were healthy men who had fathered at least one healthy child, without assisted reproductive measures during the same period as those of the cases recruited in the same hospital. A scheduled interview was arranged for each subject to collect the basic information, including personal background, lifestyle factors, occupational and environmental exposures, sexual and reproduction status, genetic risk factors, medical history and physical activity (e.g. exercise status). Then, each subject donated a 5-ml peripheral blood sample and a semen sample for genetic testing. These patients and healthy donors were all ethnically Han Chinese east China.

Semen analysis. Semen samples were obtained in private by masturbation into a sterile wide-mouth and metal-free glass container after a recommended at least 3-day sexual abstinence. After liquefaction at $37^{\circ} \mathrm{C}$ for 30 minutes, conventional semen analysis was conducted in accordance with guidelines of the WHO Laboratory Manual for the Examination of Human Semen, including semen volume, sperm number per ejaculum, sperm concentration, motility, progression and motion parameters by using Micro-cell slide and computer-aided semen analysis (CASA, WLJY 9000, Weili New Century Science and Tech Dev.). Each sample was assessed twice with strict quality control.

Variant selection and genotyping. Genomic DNA was extracted from leukocyte pellets of the venous blood by traditional proteinase $\mathrm{K}$ digestion, followed by phenol-chloroform extraction and ethanol precipitation. Genotypes were detected by the TaqMan allelic discrimination Assay on an ABI 7900HT real-time PCR system (Applied Biosystems, the United States). The gene variants studied were: DNMT1 (rs4804490); DNMT3A (rs1550117); DNMT3B (rs2424909); DNMT3L (rs7354779). We selected only variants where the frequency of the minor allele was greater than $5 \%$. For quality control, all genotyping assays were performed without knowing the case or control status of the subjects, and a random $5 \%$ of cases and controls were genotyped twice by different individuals.

Predicting the association of variant DNMT3B rs2424909, DNMT1 rs4804490, DNMT3A rs1550117 and DNMT3L rs7354779 and the binding sites of transcription factors. AliBaba2 is a program for predicting binding sites of transcription factors. We used the program to compare putative transcriptional factor-binding sites of rs2424909 and rs4804490, rs1550117 and rs7354779. Firstly, we searched the base sequences of rs 2424909 , rs4804490, rs 1550117 and rs7354779 (51 bases). Then, by respectively entering $\mathrm{C}$ allele and T allele of rs 2424909, C allele and A allele of rs4804490, A allele and G allele of rs 1550117, C allele and T allele of rs7354779, the different binding sites of transcription factors emerged.

Statistical analyses. Semen parameters were dichotomized based on WHO reference values (World Health Organization, 1999) for semen volume $(<2 \mathrm{ml})$, sperm concentration $\left(<20 \times 10^{6} / \mathrm{ml}\right)$, sperm number per ejaculate $\left(<40 \times 10^{6} / \mathrm{ml}\right)$ and sperm motility $(<50 \%$ motile sperm $)$. The control group consisted of proven fertile men with all four semen parameters at or above the WHO reference value. The subjects with idiopathic infertility were first divided into two groups according to semen parameters: case Group I was defined as idiopathic infertile men with all four semen parameters at or above the WHO reference value and the subjects in case Group II had at least one of the abnormal semen parameters. Case Group II was divided into four subgroups: Subgroup I (semen volume $<2 \mathrm{ml}$ ), Subgroup II (sperm concentration $<20 \times 10^{6} / \mathrm{ml}$ ), Subgroup III (sperm number per ejaculate $<40 \times 10^{6} / \mathrm{ml}$ ) and Subgroup IV (sperm motility $<50 \%$ motile sperm). Analysis of variance was used to compare the mean age, body mass index (BMI) and pack-years of cigarette smoking between case and control groups. The $\chi^{2}$ test was used to evaluate the difference in smoking status and alcohol status between the two groups. Multivariate logistic regression analysis was performed to obtain the odds ratios (OR) for male infertility and $95 \%$ confidence intervals (95\% CI) with adjustment for age, BMI, smoking status and alcohol drinking. All statistical analyses were carried out using Stata (Version 9.0, StataCorp LP, TX, USA), and $p$ value of less than 0.05 was considered to be statistically significant. 


\begin{tabular}{|c|c|c|c|c|c|c|c|}
\hline Characteristic & $\begin{array}{l}\text { Control } \\
(\mathrm{n}=410)^{\mathrm{a}}\end{array}$ & $\begin{array}{l}\text { Case Group I } \\
(\mathrm{n}=191)^{\mathrm{b}}\end{array}$ & $\begin{array}{l}\text { Case Group II } \\
(n=642)^{c}\end{array}$ & $\begin{array}{l}\text { Subgroup I } \\
(n=122)^{d}\end{array}$ & $\begin{array}{l}\text { Subgroup II } \\
(\mathbf{n}=340)^{\mathrm{e}}\end{array}$ & $\begin{array}{l}\text { Subgroup III } \\
(\mathrm{n}=311)^{\mathrm{f}}\end{array}$ & $\begin{array}{l}\text { Subgroup IV } \\
(\mathrm{n}=611)^{\mathrm{g}}\end{array}$ \\
\hline Age $($ mean $\pm S D)$ & $29.90 \pm 3.61$ & $29.19 \pm 3.91$ & $29.11 \pm 4.51$ & $29.39 \pm 4.86$ & $29.15 \pm 4.54$ & $29.11 \pm 4.59$ & $29.13 \pm 4.45$ \\
\hline $\mathrm{BMI}(\text { mean } \pm \mathrm{SD})^{\mathrm{h}}$ & $23.77 \pm 2.94$ & $23.23 \pm 2.92$ & $23.27 \pm 3.11$ & $23.48 \pm 3.13$ & $23.14 \pm 3.11$ & $23.12 \pm 3.12$ & $23.26 \pm 3.14$ \\
\hline \multicolumn{8}{|c|}{ Smoking status [n (\%)] } \\
\hline Never smoker & $186(45.4)$ & $84(44.0)$ & $305(47.5)$ & $48(39.3)$ & $142(41.8)$ & $127(40.8)$ & $294(48.1)$ \\
\hline Ever smoker & $224(54.6)$ & $107(56.0)$ & $337(52.5)$ & $74(60.7)$ & $198(58.2)$ & $184(59.2)$ & $317(51.9)$ \\
\hline Current smoker & $199(48.5)$ & $90(47.1)$ & $288(44.9)$ & $66(54.1)$ & $169(49.7)$ & $155(49.8)$ & $268(43.9)$ \\
\hline Former smoker & $25(6.1)$ & $17(8.9)$ & $49(7.6)$ & $8(6.6)$ & $29(8.5)$ & $29(9.3)$ & $49(8.0)$ \\
\hline $\begin{array}{l}\text { Pack-years } \\
(\text { mean } \pm \text { SD })^{\mathrm{f}}\end{array}$ & $4.58 \pm 4.70$ & $5.28 \pm 4.15$ & $5.33 \pm 5.80$ & $5.92 \pm 7.79$ & $5.30 \pm 6.04$ & $5.43 \pm 6.23$ & $5.25 \pm 5.81$ \\
\hline \multicolumn{8}{|c|}{ Drinking status [n (\%)] } \\
\hline Never drinker & $187(45.6)$ & $99(51.8)$ & $290(45.2)$ & $56(45.9)$ & $148(43.8)$ & $137(44.1)$ & $274(44.8)$ \\
\hline Ever drinker & $223(54.4)$ & $92(48.2)$ & $387(60.3)$ & $66(54.1)$ & $192(56.5)$ & $174(55.9)$ & $337(55.2)$ \\
\hline Current drinker & 211 (51.5) & $74(38.7)$ & $292(45.5)$ & $53(43.3)$ & $163(47.9)$ & $147(47.3)$ & $280(45.8)$ \\
\hline Former drinker & $12(2.9)$ & $18(9.4)$ & $95(14.8)$ & $13(10.7)$ & $29(8.5)$ & $27(8.7)$ & $57(9.3)$ \\
\hline
\end{tabular}

Table 1. Characteristics of the study population $(n=1243)$. SD, standard deviation; BMI, body mass index. aProven fertility men with semen volume $\geq 2 \mathrm{ml}$, sperm concentration $\geq 20 \times 10^{6} / \mathrm{ml}$, sperm number per ejaculum $\geq 40 \times 10^{6} / \mathrm{ml}$ and sperm motility $\geq 50 \%$ motile sperm. ${ }^{b}$ Idiopathic infertile men with all four semen parameters at or above the WHO reference value. 'Idiopathic infertile men with at least one of the abnormal semen parameters (semen volume, sperm concentration, sperm number per ejaculum and sperm motility). ${ }^{\mathrm{d} I d i o p a t h i c ~ i n f e r t i l e ~ m e n ~ w i t h ~ s e m e n ~ v o l u m e ~}<2 \mathrm{ml}$. Idiopathic infertile men with sperm concentration $<20 \times 10^{6} / \mathrm{ml}$. Idiopathic infertile men with sperm number per ejaculum $<40 \times 10^{6} / \mathrm{ml}$. ${ }^{\text {g Idiopathic infertile }}$ men with sperm motility $<50 \%$ motile sperm. ${ }^{\mathrm{h} B M I}: \mathrm{kg} / \mathrm{m}^{2}$.

\section{Results}

Characteristics of the study population. The final study consisted of 1243 men, 833 idiopathic infertile patients and 410 fertile controls. All subjects were Han Chinese, with a mean $( \pm$ SD) age of $29.38( \pm 4.15)$ years. Eight hundred and thirty three men were idiopathic infertile men, 191 men (22.9\%; Case Group I) with normal semen quality and 642 men (77.1\%; Case Group II) with abnormal semen quality according to WHO standard. In addition, patients in the Case Group II were further divided into four subgroups: Subgroup I: patients with semen volume $<2 \mathrm{ml}(\mathrm{n}=122)$; Subgroup II: subjects with sperm concentration $<20 \times 10^{6} / \mathrm{ml}(\mathrm{n}=340)$; Subgroup III: patients with sperm number per ejaculum $<40 \times 10^{6} / \mathrm{ml}(\mathrm{n}=311)$; and Subgroup IV: patients with sperm motility $<50 \%$ motile sperm $(n=611)$. Demographic categories by fertility and semen quality are described in Table 1 . Infertile males were younger, had a lower BMI and were more likely to be former drinkers than the fertile controls (Table 1). All variables were further adjusted for any residue confounding effect in later multivariate logistic regression analyses.

Association between genotype distributions of four DNA methyltransferases and semen quality. The genotype distributions of the four DNMT genes among the cases and controls are shown in Tables 2 and 3. As shown in Table 2, we initially divided the cases into the Case Group I and Case Group II according to semen parameters. When the DNMT1 CC genotype was used as the reference group, the CA genotype was significantly associated with increased risk for male infertility in Case Group I (adjusted OR, 1.50, 95\% CI $=1.00-2.25$, $P=0.048$ ) and Case Group II (adjusted OR, 1.40, 95\% CI $=1.05-1.86, P=0.023$ ). Under the recessive model of inheritance, the DNMT1 AA genotype was associated with significantly decreased risk for male infertility with abnormal semen parameters (adjusted OR, 0.68, 95\% CI $=0.50-0.92, P=0.012$ ), compared with other genotypes. In addition, compared to the DNMT3B CC genotype, the association between the CT genotype and male infertility was not significantly different (adjusted $\mathrm{OR}, 1.25,95 \% \mathrm{CI}=0.85-1.83, P=0.255$ ), but the TT homozygous genotype was associated with significantly decreased risk for male infertility with abnormal semen parameters (adjusted OR, 0.25, 95\% CI $=0.06-0.94, P=0.040$ ). Under the recessive model of inheritance, the DNMT3B variant homozygote was associated with significantly decreased risk for male infertility with abnormal semen parameters (adjusted $\mathrm{OR}, 0.24,95 \% \mathrm{CI}=0.06-0.90, P=0.034$ ), compared with other genotypes, after adjustment for age, BMI, smoking and drinking status in the multivariate logistic regression analysis (Table 2). In contrast, no significant differences were found for rs 1550117 and rs7354779 between the cases and controls.

In order to further assess the possibility of an association between the four DNMTs genetic variants and a particular aspect of semen parameters, the patients in case Group II were further stratified into four subgroups on the basis of semen volume, sperm concentration, sperm number per ejaculum or sperm motility as described in the section of statistical analysis. As illustrated in Table 3, subjects in Subgroup II and Subgroup IV had a significantly increased frequency of the heterozygous DNMT1 CA genotype compared with fertile controls (adjusted OR, 1.43, 95\% CI $=1.02-1.99, P=0.037$ for Subgroup II; adjusted OR, $1.40,95 \%$ CI $=1.05-1.86$, $P=0.023$ for Subgroup IV). Under the recessive model of inheritance, subjects in Subgroup IV had a significantly decreased frequency of the DNMT1 variant homozygote compared with other genotypes (adjusted OR, 0.68, $95 \% \mathrm{CI}=0.50-0.92, P=0.014)$. When the DNMT3B CC genotype was used as the reference group, the carrier of TT genotype in Subgroup IV had a significantly decreased risk of male infertility (adjusted OR, 0.26, 95\% 


\begin{tabular}{|c|c|c|c|c|c|c|c|c|}
\hline \multirow[b]{2}{*}{ SNPs } & \multirow[b]{2}{*}{ Genotype } & \multirow{2}{*}{$\begin{array}{l}\text { Control }(\mathrm{n}=410)^{\mathrm{a}} \\
\mathrm{n}(\%)\end{array}$} & \multicolumn{3}{|c|}{ Case Group I $(\mathrm{n}=191)^{\mathrm{b}}$} & \multicolumn{3}{|c|}{ Case Group II $(n=642)^{c}$} \\
\hline & & & n (\%) & $P^{\mathrm{d}, \mathrm{e}}$ & OR $(95 \% \mathrm{CI})^{\mathrm{d}, \mathrm{f}}$ & n (\%) & $P^{\mathrm{d}, \mathrm{e}}$ & OR $(95 \% \mathrm{CI})^{\mathrm{d}, \mathrm{f}}$ \\
\hline \multirow{5}{*}{ rs 4804490} & $\mathrm{CC}$ & $144(35.3)$ & $55(29.3)$ & & 1.00 (reference) & $204(31.9)$ & & 1.00 (reference) \\
\hline & CA & $166(40.7)$ & $96(51.1)$ & 0.048 & $1.50(1.00-2.25)$ & $324(50.6)$ & 0.023 & $1.40(1.05-1.86)$ \\
\hline & AA & $98(24.0)$ & $37(19.7)$ & 0.985 & $1.00(0.61-1.63)$ & $112(17.5)$ & 0.281 & $0.82(0.58-1.17)$ \\
\hline & C-allele carriers & $310(76.0)$ & $151(80.3)$ & & 1.00 (reference) & $528(82.5)$ & & 1.00 (reference) \\
\hline & AA & $98(24.0)$ & $37(19.7)$ & 0.294 & $0.79(0.52-1.22)$ & $112(17.5)$ & 0.012 & $0.68(0.50-0.92)$ \\
\hline \multirow{5}{*}{ rs 1550117} & GG & $263(64.8)$ & $115(60.2)$ & & 1.00 (reference) & $405(64.0)$ & & 1.00 (reference) \\
\hline & GA & $122(30.0)$ & $70(36.6)$ & 0.163 & $1.30(0.90-1.89)$ & $201(31.8)$ & 0.829 & $1.03(0.78-1.36)$ \\
\hline & AA & $21(5.2)$ & $6(3.1)$ & 0.452 & $0.69(0.27-1.80)$ & $27(4.3)$ & 0.476 & $0.80(0.44-1.46)$ \\
\hline & G-allele carriers & $385(94.8)$ & $185(96.9)$ & & 1.00 (reference) & $606(95.7)$ & & 1.00 (reference) \\
\hline & AA & $21(5.2)$ & $6(3.1)$ & 0.315 & $0.62(0.24-1.58)$ & $27(4.3)$ & 0.457 & $0.80(0.44-1.44)$ \\
\hline \multirow{5}{*}{ rs2424909 } & $\mathrm{CC}$ & $344(86.4)$ & $160(83.8)$ & & 1.00 (reference) & $537(84.8)$ & & 1.00 (reference) \\
\hline & CT & $46(11.6)$ & $30(15.7)$ & 0.246 & $1.35(0.81-2.23)$ & $93(14.7)$ & 0.255 & $1.25(0.85-1.83)$ \\
\hline & TT & $8(2.0)$ & $1(0.5)$ & 0.242 & $0.29(0.04-2.32)$ & $3(0.5)$ & 0.040 & $0.25(0.06-0.94)$ \\
\hline & C-allele carriers & $390(98.0)$ & $190(99.5)$ & & 1.00 (reference) & $630(99.5)$ & & 1.00 (reference) \\
\hline & TT & $8(2.0)$ & $1(0.5)$ & 0.229 & $0.28(0.03-2.25)$ & $3(0.5)$ & 0.034 & $0.24(0.06-0.90)$ \\
\hline \multirow{5}{*}{ rs7354779 } & TT & $384(93.7)$ & $177(95.2)$ & & 1.00 (reference) & $597(94.1)$ & & 1.00 (reference) \\
\hline & TC & $24(5.9)$ & $9(4.8)$ & 0.555 & $0.79(0.35-1.75)$ & $36(5.7)$ & 0.978 & $0.99(0.58-1.70)$ \\
\hline & CC & $2(0.5)$ & $0(0)$ & - & - & $2(0.3)$ & 0.673 & $0.65(0.09-4.73)$ \\
\hline & T-allele carriers & 408 (99.5) & $186(100)$ & & 1.00 (reference) & $633(99.7)$ & & 1.00 (reference) \\
\hline & $\mathrm{CC}$ & $2(0.5)$ & $0(0)$ & 0.593 & $0.88(0.55-1.41)$ & $2(0.3)$ & 0.675 & $0.65(0.09-4.74)$ \\
\hline
\end{tabular}

Table 2. Genotype frequencies of DNMTs genetic variants among the cases and controls and their association with male infertility. SNPs, single-nucleotide polymorphisms. OR, odds ratios; CI, confidence interval. ${ }^{\text {a Proven }}$ fertility men with semen volume $\geq 2 \mathrm{ml}$, sperm concentration $\geq 20 \times 10^{6} / \mathrm{ml}$, sperm number per ejaculum $\geq 40 \times 10^{6} / \mathrm{ml}$ and sperm motility $\geq 50 \%$ motile sperm. ${ }^{b}$ Idiopathic infertile men with all four semen parameters at or above the WHO reference value. 'Idiopathic infertile men with at least one of the abnormal semen parameters (semen volume, sperm concentration, sperm number per ejaculum and sperm motility). ${ }^{\mathrm{d}}$ Adjusted for age, BMI, smoking status and drinking status. ${ }^{\text {TTwo-sided }} \chi^{2}$ test for genotype distributions between cases and controls. ${ }^{\mathrm{f} O R s}$ were obtained from multivariate logistic regression analysis.

$\mathrm{CI}=0.07-0.98, P=0.046)$. The $D N M T 3 B$ variant homozygote had a significantly decreased risk of male infertility in the Subgroup IV (adjusted OR, 0.25, 95\% CI =0.06-0.94, $P=0.040$ ) compared with other genotypes (Table 3). In contrast, no significant differences were found for DNMT3A (rs1550117) and DNMT3L (rs7354779) between the cases and controls (Table 3 ).

Effects of the variant rs2424909 and rs4804490 on the binding of transcription factors. According to AliBaba2 programs, with the transition of "C" to "T" of the variant DNMT3B rs2424909 allele, new binding sites for transcription factors (Figure S1) were generated. Figure S2 indicated that the transition of "C" to "A" of the variant DNMT1 rs4804490 allele generated new binding sites for transcription factors. Figures S3 and S4 showed that the transition of " $\mathrm{A}$ " to " $\mathrm{G}$ " of the variant DNMT3A rs 1550117 allele and "C" to "T" of the variant DNMT3L rs7354779 generated new binding sites for transcription factors.

\section{Discussion}

Several studies have revealed the close relationship of DNMTs with the male reproductive system, and potential associations with human infertility. Although it has been identified that DNMTs play critical roles in spermatogenesis and male fertility, a genetic study of four DNMTs in idiopathic male infertility has not been performed yet.

This case-control study analyzed DNMTs polymorphisms to define their association with semen parameters and male infertility. We have demonstrated a significantly increased risk of idiopathic male infertility with abnormal semen parameters in association with heterozygote of variant rs 4804490 , and a decreased risk of idiopathic infertility with abnormal semen parameters in association with variant homozygote of variant rs2424909 $(P=0.023$ and 0.040 , respectively). In addition, under the recessive model of inheritance, the AA genotype of variant rs4804490 and TT genotype of variant rs2424909 were significantly associated with decreased risk for male infertility with abnormal semen parameters. Among men with normal semen parameters, there were no significant differences in risks associated with these genotypes. Furthermore, no significant associations with risk of idiopathic infertility associated with either normal or abnormal semen parameters were found for the rs 1550117 and rs7354779 variants. A recent study by Huang et al. investigated the association between DNMT3L polymorphisms and male infertility with azoospermia ${ }^{33}$. They found that the presence of rs2070565 allele A increased the risk of azoospermia in males. Additionally, they did not report any significant difference for rs 7354779 between the infertile patients and fertile controls, which similar to our findings. These results suggest that different variants in the genes involved in epigenetic pathways may have different relationships with idiopathic male infertility and 


\begin{tabular}{|c|c|c|c|c|c|c|c|c|c|c|c|c|c|c|}
\hline \multirow[b]{2}{*}{ SNPs } & \multirow[b]{2}{*}{ Genotype } & \multirow{2}{*}{\begin{tabular}{|l|}
$\begin{array}{l}\text { Control } \\
(\mathrm{n}=410)^{\mathrm{a}}\end{array}$ \\
$\mathrm{n}(\%)$ \\
\end{tabular}} & \multicolumn{3}{|c|}{ Subgroup I $(n=122)^{b, f}$} & \multicolumn{3}{|c|}{ Subgroup II $(n=340)^{c, f}$} & \multicolumn{3}{|c|}{ Subgroup III $(n=311)^{\mathrm{d}, \mathrm{f}}$} & \multicolumn{3}{|c|}{ Subgroup IV $(n=611)^{e, f}$} \\
\hline & & & n (\%) & $P g, \mathrm{~h}$ & OR $(95 \% \mathrm{CI})^{\mathrm{g}, \mathrm{i}}$ & n (\%) & $P$ g,h & OR $(95 \% \mathrm{CI})^{\mathrm{g}, \mathrm{i}}$ & $\mathbf{n}(\%)$ & $P g, h$ & OR $(95 \% \text { CI })^{g, i}$ & n (\%) & $P$ g,h & OR $(95 \% \mathrm{CI})^{\mathrm{g}, \mathrm{i}}$ \\
\hline \multirow{5}{*}{ rs 4804490} & CC & $144(35.3)$ & $42(34.7)$ & & 1.00 (reference) & $108(31.9)$ & & 1.00 (reference) & $102(32.9)$ & & 1.00 (reference) & $193(31.7)$ & & 1.00 (reference) \\
\hline & $\mathrm{CA}$ & $166(40.7)$ & $55(45.4)$ & 0.525 & $1.16(0.73-1.84)$ & $170(50.1)$ & 0.037 & $1.43(1.02-1.99)$ & $151(48.7)$ & 0.084 & $1.35(0.96-1.90)$ & $309(50.7)$ & 0.023 & $1.40(1.05-1.86)$ \\
\hline & AA & $98(24.0)$ & $24(19.8)$ & 0.629 & $0.87(0.49-1.54)$ & $61(18.0)$ & 0.606 & $0.90(0.59-1.36)$ & $57(18.4)$ & 0.624 & $0.90(0.59-1.38)$ & 107 (17.6) & 0.287 & $0.83(0.58-1.18)$ \\
\hline & C-allele carriers & $310(76.0)$ & $97(80.2)$ & & 1.00 (reference) & $278(82.0)$ & & 1.00 (reference) & $253(81.6)$ & & 1.00 (reference) & $502(82.4)$ & & 1.00 (reference) \\
\hline & AA & $98(24.0)$ & $24(19.8)$ & 0.357 & $0.79(0.48-1.31)$ & $61(18.0)$ & 0.066 & $0.71(0.50-1.02)$ & $57(18.4)$ & 0.099 & $0.73(0.51-1.06)$ & $107(17.6)$ & 0.014 & $0.68(0.50-0.92)$ \\
\hline \multirow{5}{*}{ rs1550117 } & GG & $263(64.8)$ & $82(67.2)$ & & 1.00 (reference) & $215(64.2)$ & & 1.00 (reference) & $193(63.1)$ & & 1.00 (reference) & $381(63.3)$ & & 1.00 (reference) \\
\hline & GA & $122(30.0)$ & $34(27.9)$ & 0.600 & $0.89(0.56-1.40)$ & $104(31.0)$ & 0.964 & $1.01(0.73-1.39)$ & $100(32.7)$ & 0.597 & $1.09(0.79-1.52)$ & $196(32.6)$ & 0.645 & $1.07(0.81-1.41)$ \\
\hline & AA & $21(5.2)$ & $6(4.9)$ & 0.842 & $0.91(0.35-2.35)$ & $16(4.8)$ & 0.806 & $0.92(0.46-1.83)$ & $13(4.2)$ & 0.618 & $0.83(0.40-1.73)$ & $25(4.2)$ & 0.488 & $0.81(0.44-1.48)$ \\
\hline & G-allele carriers & $385(94.8)$ & $116(95.1)$ & & 1.00 (reference) & 319 (93.2) & & 1.00 (reference) & $293(95.8)$ & & 1.00 (reference) & $577(95.8)$ & & 1.00 (reference) \\
\hline & AA & $21(5.2)$ & $6(4.9)$ & 0.911 & $0.95(0.37-2.42)$ & $16(4.8)$ & 0.770 & $0.90(0.46-1.78)$ & $13(4.2)$ & 0.527 & $0.79(0.38-1.63)$ & $25(4.2)$ & 0.453 & $0.79(0.43-1.45)$ \\
\hline \multirow{5}{*}{ rs2424909 } & $\mathrm{CC}$ & $344(86.4)$ & $102(85.0)$ & & 1.00 (reference) & $290(86.6)$ & & 1.00 (reference) & $264(86.3)$ & & 1.00 (reference) & 509 (84.6) & & 1.00 (reference) \\
\hline & CT & $46(11.6)$ & $18(15.0)$ & 0.383 & $1.30(0.72-2.36)$ & $43(12.8)$ & 0.792 & $1.06(0.68-1.67)$ & $40(13.1)$ & 0.703 & $1.09(0.69-1.73)$ & $90(15.0)$ & 0.220 & $1.27(0.87-1.87)$ \\
\hline & TT & $8(2.0)$ & $0(0.0)$ & 0.980 & $1.00(0.82-1.23)$ & $2(0.6)$ & 0.145 & $0.31(0.06-1.50)$ & $2(0.7)$ & 0.185 & $0.35(0.07-1.66)$ & $3(0.5)$ & 0.046 & $0.26(0.07-0.98)$ \\
\hline & C-allele carriers & $390(98.0)$ & $120(100.0)$ & & 1.00 (reference) & 333 (99.4) & & 1.00 (reference) & 304 (99.3) & & 1.00 (reference) & $599(99.5)$ & & 1.00 (reference) \\
\hline & TT & $8(2.0)$ & $0(0.0)$ & 0.984 & $1.00(0.82-1.23)$ & $2(0.6)$ & 0.138 & $0.31(0.06-1.47)$ & $2(0.7)$ & 0.175 & $0.34(0.07-1.62)$ & $3(0.5)$ & 0.040 & $0.25(0.06-0.94)$ \\
\hline \multirow{5}{*}{ rs7354779 } & TT & $384(93.7)$ & $110(91.7)$ & & 1.00 (reference) & $311(92.6)$ & & 1.00 (reference) & $282(91.9)$ & & 1.00 (reference) & 567 (93.9) & & 1.00 (reference) \\
\hline & $\mathrm{TC}$ & $24(5.9)$ & $9(7.5)$ & 0.529 & $1.29(0.58-2.87)$ & $24(7.1)$ & 0.443 & $1.26(0.70-2.27)$ & $24(7.8)$ & 0.273 & $1.39(0.77-2.52)$ & $36(6.0)$ & 0.862 & $1.05(0.61-1.79)$ \\
\hline & $\mathrm{CC}$ & $2(0.5)$ & $1(0.8)$ & 0.640 & $1.79(0.16-20.28)$ & $1(0.3)$ & 0.632 & $0.55(0.05-6.52)$ & $1(0.3)$ & 0.701 & $0.61(0.05-7.40)$ & $1(0.2)$ & 0.324 & $0.29(0.02-3.44)$ \\
\hline & T-allele carriers & 408 (99.5) & $119(99.2)$ & & 1.00 (reference) & 335 (99.7) & & 1.00 (reference) & 306 (99.7) & & 1.00 (reference) & $603(99.8)$ & & 1.00 (reference) \\
\hline & $\mathrm{CC}$ & $2(0.5)$ & $1(0.8)$ & 0.675 & $1.68(0.15-19.13)$ & $1(0.3)$ & 0.626 & $0.54(0.04-6.46)$ & $1(0.3)$ & 0.690 & $0.60(0.05-7.27)$ & $1(0.2)$ & 0.325 & $0.29(0.02-3.45)$ \\
\hline
\end{tabular}

Table 3. Genotype frequencies of DNMTs genetic variants among the cases and controls and their association with male infertility. SNPs, single-nucleotide polymorphisms. OR, odds ratios; CI, confidence interval. ${ }^{\text {a Proven }}$ fertility men with semen volume $\geq 2 \mathrm{ml}$, sperm concentration $\geq 20 \times 10^{6} / \mathrm{ml}$, sperm number per ejaculum

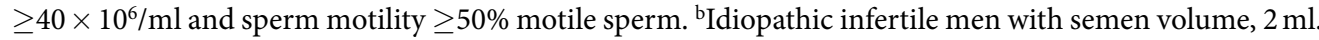
${ }^{c}$ Idiopathic infertile men with sperm concentration, $20 \times 10^{6} / \mathrm{ml}$. ${ }^{\mathrm{d}}$ Idiopathic infertile men with sperm number per ejaculum, $40 \times 10^{6} / \mathrm{ml}$. ${ }^{\mathrm{e}}$ Idiopathic infertile men with sperm motility, $50 \%$ motile sperm. ${ }^{\text {fThe semen }}$ parameter categories were not mutually exclusive; a subject may contribute data to more than one category. ${ }^{\mathrm{g}}$ Adjusted for age, BMI, smoking status and drinking status. ${ }^{\mathrm{h}}$ Two-sided $\chi^{2}$ test for genotype distributions between cases and controls. ${ }^{\mathrm{I}}$ ORs were obtained from multivariate logistic regression analysis.

men with these variants have an increased or increased risk of abnormal semen parameters associated with male infertility.

Genetic disruption of both DNMT1 and DNMT3B nearly eliminated methyltransferase activity, and reduced genomic DNA methylation by greater than $95 \%{ }^{34}$. These marked changes resulted in demethylation of repeated sequences, loss of insulin-like growth factor II (IGF2) imprinting, abrogation of silencing of the tumor suppressor gene $\mathrm{p} 16$, and growth suppression ${ }^{34}$. Rhee and colleagues demonstrated that these two enzymes cooperatively maintain DNA methylation and gene silencing in human cancer cells ${ }^{34}$. The precise mechanism of the variant rs4804490 and rs2424909 in male infertility remains unclear, as there is no direct functional data available. A single-base substitution may change the binding of transcription factors and consequently influence their function. We therefore raised hypotheses that these variations may be associated with changes of transcription factors. Changes of the binding of transcription factors were observed with both of the variations. Variants of DNMT1 and DNMT3B are risk factors for idiopathic male infertility.

In conclusion, our study demonstrates that some representative variants of the DNMT genes may modulate the risk of male infertility associated with abnormal semen parameters. Confirmation of similar findings in larger study groups would be needed. In summary, in this case-control study, we found that the carrier of DNMT1 CA genotype had a significantly increased risk of idiopathic male infertility compared with DNMT1 CC genotype carriers, although no significant difference was found in DNMT3A and DNMT3L. Our results support the hypothesis that DNMT1 polymorphism may be associated with an increased risk of idiopathic male infertility in a Han-Chinese population. These SNPs may alter a transcription factor binding site in the alternative promoter, and the variant genotype (s) may be in linkage disequilibrium with other untyped susceptibility loci. Moreover, the variants may alter the binding of a regulatory miRNA or contribute to the differential expression of alternatively spliced DNMT3B variants.

As shown in Fig. S1, the rs2424909 C > T variation creates a binding site for transcription factors GR, and deletes two binding sites for ADR1 and AP-2alph. GR is a ligand-activated transcription factor. In addition to activating enhancers harboring glucocorticoid response elements (GREs), it also inhibits the actions of other transcription factors, including AP1 and NF- $\kappa$ B. In ER $\alpha$-positive breast cancer, GR expression has been associated with good clinical outcomes ${ }^{35,36}$. AP-1 transcription factor, known to play a vital role in cell proliferation and neuronal activation, is also involved in cell apoptosis in response to stress, lack of survival signals or DNA damaging agents ${ }^{37}$. Nuclear factor- $\kappa \mathrm{B}(\mathrm{NF}-\kappa \mathrm{B})$ transcription factor regulates a wide array of genes mediating numerous 
cellular processes such as proliferation, differentiation, motility and survival ${ }^{38}$. In Fig. S2, rs4804490 C $>$ A variation creates two binding sites for transcription factors NF-1 and CTF, deleting two binding sites for c-Jun and $\mathrm{Sp} 1$. The CAAT box-binding transcription factor/nuclear factor-1 (NF1, also called CTF/NF1) consists of widely expressed transcription factors, comprising NF1-A, NF1-B, NF1-C and NF1-X subtypes ${ }^{39,40}$. Transcription factor Specificity protein 1 (Sp1) plays a role in promoting oncogenes required for tumor survival, metastasis and progression. c-Jun, a component of the transcription factor AP-1, regulates gene expression and cell function.

At present, there is no evidence that these transcription factors interact with DNMTs to control their DNA binding specificity. We suggest that the genetic variants DNMT3B and DNMT1 could regulate gene expression by the creation or deletion of transcription factors and influence the sperm maturation process and male fertility. Microarray analysis and real time quantitative PCR has shown that the differential gene expression of DNMT1 between various forms of testicular cancer was consistent ${ }^{41}$. Adiga et al. documented that DNMT3B is differentially expressed ${ }^{42}$ in the preleptotene/zygotene and pachytene spermatocytes from fertile and infertile men. Future mechanistic studies about functional significance of the variation at DNMT1 and DNMT3B will help fully flesh out the relationship between polymorphism of DNMTs, abnormal DNA methylation patterns, and spermatogenic failure.

\section{References}

1. Guzick, D. S. et al. Sperm morphology, motility, and concentration in fertile and infertile men. N Engl J Med 345, 1388-1393, doi:https://doi.org/10.1056/NEJMoa003005 (2001).

2. Okada, H. et al. Genome-wide expression of azoospermia testes demonstrates a specific profile and implicates ART3 in genetic susceptibility. PLoS genetics 4, e26, doi:https://doi.org/10.1371/journal.pgen.0040026 (2008).

3. Lande-Diner, L. et al. Role of DNA methylation in stable gene repression. The Journal of biological chemistry 282, 12194-12200, doi:https://doi.org/10.1074/jbc.M607838200 (2007).

4. Du, Y. et al. Promoter targeted bisulfite sequencing reveals DNA methylation profiles associated with low sperm motility in asthenozoospermia. Hum Reprod 31, 24-33, doi:https://doi.org/10.1093/humrep/dev283 (2016).

5. Jenkins, T. G. et al. Decreased fecundity and sperm DNA methylation patterns. Fertil Steril 105, 51-57 e51-53, doi:https://doi. org/10.1016/j.fertnstert.2015.09.013 (2016).

6. Zhou, J. H. et al. The expression of cysteine-rich secretory protein 2 (CRISP2) and its specific regulator miR-27b in the spermatozoa of patients with asthenozoospermia. Biol Reprod 92, 28, doi:https://doi.org/10.1095/biolreprod.114.124487 (2015).

7. Li, L. et al. Whole-exome sequencing identified a homozygous BRDT mutation in a patient with acephalic spermatozoa. Oncotarget. doi:https://doi.org/10.18632/oncotarget.15251 (2017).

8. Zhang, S. et al. Association between DAZL polymorphisms and susceptibility to male infertility: systematic review with metaanalysis and trial sequential analysis. Sci Rep 4, 4642, doi:https://doi.org/10.1038/srep04642 (2014).

9. Bourc'his, D. \& Bestor, T. H. Meiotic catastrophe and retrotransposon reactivation in male germ cells lacking Dnmt3L. Nature 431, 96-99, doi:https://doi.org/10.1038/nature02886 (2004)

10. Kaneda, M. et al. Essential role for de novo DNA methyltransferase Dnmt3a in paternal and maternal imprinting. Nature 429, 900-903, doi:https://doi.org/10.1038/nature02633 (2004).

11. Kato, Y. et al. Role of the Dnmt3 family in de novo methylation of imprinted and repetitive sequences during male germ cell development in the mouse. Hum Mol Genet 16, 2272-2280, doi:https://doi.org/10.1093/hmg/ddm179 (2007).

12. Takashima, S. et al. Abnormal DNA methyltransferase expression in mouse germline stem cells results in spermatogenic defects. Biol Reprod 81, 155-164, doi:https://doi.org/10.1095/biolreprod.108.074708 (2009).

13. Jones, P. A. \& Baylin, S. B. The fundamental role of epigenetic events in cancer. Nat Rev Genet 3, 415-428, doi:https://doi. org/10.1038/nrg816 (2002).

14. Frigola, J. et al. Epigenetic remodeling in colorectal cancer results in coordinate gene suppression across an entire chromosome band. Nat Genet 38, 540-549, doi:https://doi.org/10.1038/ng1781 (2006).

15. Cebrian, A. et al. Genetic variants in epigenetic genes and breast cancer risk. Carcinogenesis 27, 1661-1669, doi:https://doi. org/10.1093/carcin/bgi375 (2006)

16. Bestor, T. H. Cytosine methylation and the unequal developmental potentials of the oocyte and sperm genomes. Am J Hum Genet 62, 1269-1273, doi:https://doi.org/10.1086/301891 (1998).

17. Okano, M., Xie, S. \& Li, E. Dnmt2 is not required for de novo and maintenance methylation of viral DNA in embryonic stem cells. Nucleic Acids Res 26, 2536-2540, doi:gkb442 (1998).

18. Okano, M., Bell, D. W., Haber, D. A. \& Li, E. DNA methyltransferases Dnmt3a and Dnmt3b are essential for de novo methylation and mammalian development. Cell 99, 247-257, doi:S0092-8674(00)81656-6 (1999).

19. Xie, S. et al. Cloning, expression and chromosome locations of the human DNMT3 gene family. Gene 236, 87-95, doi:S0378-1119(99)00252-8 (1999).

20. Aapola, U. et al. Isolation and initial characterization of a novel zinc finger gene, DNMT3L, on 21q22.3, related to the cytosine-5methyltransferase 3 gene family. Genomics 65, 293-298, doi:https://doi.org/10.1006/geno.2000.6168 (2000).

21. Webster, K. E. et al. Meiotic and epigenetic defects in Dnmt3L-knockout mouse spermatogenesis. Proc Natl Acad Sci USA 102, 4068-4073, doi:https://doi.org/10.1073/pnas.0500702102 (2005).

22. Chen, Z. X. \& Riggs, A. D. Maintenance and regulation of DNA methylation patterns in mammals. Biochem Cell Biol 83, 438-448, doi:https://doi.org/10.1139/o05-138 (2005).

23. Chen, T. \& Li, E. Establishment and maintenance of DNA methylation patterns in mammals. Curr Top Microbiol Immunol 301, 179-201 (2006)

24. Goll, M. G. \& Bestor, T. H. Eukaryotic cytosine methyltransferases. Annu Rev Biochem 74, 481-514, doi:https://doi.org/10.1146/ annurev.biochem.74.010904.153721 (2005).

25. Chedin, F., Lieber, M. R. \& Hsieh, C. L. The DNA methyltransferase-like protein DNMT3L stimulates de novo methylation by Dnmt3a. Proc Natl Acad Sci USA 99, 16916-16921, doi:https://doi.org/10.1073/pnas.262443999 (2002).

26. Jia, D., Jurkowska, R. Z., Zhang, X., Jeltsch, A. \& Cheng, X. Structure of Dnmt3a bound to Dnmt3L suggests a model for de novo DNA methylation. Nature 449, 248-251, doi:https://doi.org/10.1038/nature06146 (2007).

27. Kareta, M. S., Botello, Z. M., Ennis, J. J., Chou, C. \& Chedin, F. Reconstitution and mechanism of the stimulation of de novo methylation by human DNMT3L. J Biol Chem 281, 25893-25902, doi:https://doi.org/10.1074/jbc.M603140200 (2006).

28. Li, E., Bestor, T. H. \& Jaenisch, R. Targeted mutation of the DNA methyltransferase gene results in embryonic lethality. Cell 69, 915-926, doi:0092-8674(92)90611-F (1992).

29. Bourc'his, D., Xu, G. L., Lin, C. S., Bollman, B. \& Bestor, T. H. Dnmt3L and the establishment of maternal genomic imprints. Science 294, 2536-2539, doi:https://doi.org/10.1126/science.1065848 (2001).

30. Robertson, K. D. DNA methylation and human disease. Nat Rev Genet 6, 597-610, doi:https://doi.org/10.1038/nrg1655 (2005)

31. Richardson, B. DNA methylation and autoimmune disease. Clin Immunol 109, 72-79, doi:S1521661603002067 (2003). 
32. Feinberg, A. P. \& Tycko, B. The history of cancer epigenetics. Nat Rev Cancer 4, 143-153, doi:https://doi.org/10.1038/nrc1279 (2004).

33. Huang, J. X., Scott, M. B., Pu, X. Y. \& A, Z. C. Association between single-nucleotide polymorphisms of DNMT3L and infertility with azoospermia in Chinese men. Reprod Biomed Online, doi:https://doi.org/10.1016/j.rbmo.2011.09.004 (2011).

34. Rhee, I. et al. DNMT1 and DNMT3b cooperate to silence genes in human cancer cells. Nature 416, 552-556, doi:https://doi. org/10.1038/416552a (2002).

35. Abduljabbar, R. et al. Clinical and biological significance of glucocorticoid receptor (GR) expression in breast cancer. Breast cancer research and treatment 150, 335-346, doi:https://doi.org/10.1007/s10549-015-3335-1 (2015).

36. Pan, D., Kocherginsky, M. \& Conzen, S. D. Activation of the glucocorticoid receptor is associated with poor prognosis in estrogen receptor-negative breast cancer. Cancer research 71, 6360-6370, doi:https://doi.org/10.1158/0008-5472.CAN-11-0362 (2011).

37. Kaminska, B., Pyrzynska, B., Ciechomska, I. \& Wisniewska, M. Modulation of the composition of AP-1 complex and its impact on transcriptional activity. Acta neurobiologiae experimentalis 60, 395-402 (2000).

38. Soubannier, V. \& Stifani, S. NF-kappaB Signalling in Glioblastoma. Biomedicines 5, doi:https://doi.org/10.3390/biomedicines5020029 (2017).

39. Rupp, R. A. et al. Chicken NFI/TGGCA proteins are encoded by at least three independent genes: NFI-A, NFI-B and NFI-C with homologues in mammalian genomes. Nucleic acids research 18, 2607-2616 (1990).

40. Kruse, U., Qian, F. \& Sippel, A. E. Identification of a fourth nuclear factor I gene in chicken by cDNA cloning: NFI-X. Nucleic acids research 19, 6641 (1991).

41. Omisanjo, O. A. et al. DNMT1 and HDAC1 gene expression in impaired spermatogenesis and testicular cancer. Histochemistry and cell biology 127, 175-181, doi:https://doi.org/10.1007/s00418-006-0234-x (2007).

42. Adiga, S. K. et al. Reduced expression of DNMT3B in the germ cells of patients with bilateral spermatogenic arrest does not lead to changes in the global methylation status. Molecular human reproduction 17, 545-549, doi:https://doi.org/10.1093/molehr/gar023 (2011).

\section{Acknowledgements}

This work was supported by National Natural Science Foundation of China (Nos 81302457, 81673217, 81401213), National Key Research and Development Program (2016YFC1000207), and the Priority Academic Program for the Development of Jiangsu Higher Education Institutions (Public Health and Preventive Medicine).

\section{Author Contributions}

Conceived and designed the experiments: W.W., Q.T., Y.C. Conducted the experiments: Q.T., Y.C., Analyzed the data: W.W., Q.T., Y.C. Prepare figures: W.W., Q.T., Y.C. Contributed materials/analysis tools: W.W., Y.X., H.D., D.C., X.W. Wrote and revised the paper: W.W., Q.T., YC.

\section{Additional Information}

Supplementary information accompanies this paper at doi:10.1038/s41598-017-11636-9

Competing Interests: The authors declare that they have no competing interests.

Publisher's note: Springer Nature remains neutral with regard to jurisdictional claims in published maps and institutional affiliations.

(c) (i) Open Access This article is licensed under a Creative Commons Attribution 4.0 International License, which permits use, sharing, adaptation, distribution and reproduction in any medium or format, as long as you give appropriate credit to the original author(s) and the source, provide a link to the Creative Commons license, and indicate if changes were made. The images or other third party material in this article are included in the article's Creative Commons license, unless indicated otherwise in a credit line to the material. If material is not included in the article's Creative Commons license and your intended use is not permitted by statutory regulation or exceeds the permitted use, you will need to obtain permission directly from the copyright holder. To view a copy of this license, visit http://creativecommons.org/licenses/by/4.0/.

(c) The Author(s) 2017 